Z. Klin. Chem. Klin. Biochem.

11. Jg. 1973 , S. $543-547$

\title{
Die Bedeutung von Membranphospholipiden für Antigen-Antikörper-Bindung im Rh-D-System1)
}

\author{
Von H. Weicker, H. Schmid, J. Kropp, V. Helmstädter und W. Ejert \\ Alus der S.J. Thannbauser-Abteilung fïr Stoffwechseluntersucbungen (Vorstand: Prof. Dr. H. Weicker) \\ der Mediziniscben Uniw.-Poliklinik Heidelberg
}

(Eingegangen am 28. August/8. Oktober 1973)

\begin{abstract}
Die $\mathrm{Rh}$-D-Antigenität eines gereinigten Membranproteins von Rh-positiven Erythrocyten wurde nach aktiver und passiver Immunisierung von Mecrschweinchen in dem SchuLrz-DALE-Versuch an der glatten Uterusmuskulatur nachgewiesen. Nach Phospholipase AInkubation des 'Testmuskels war keine Antigen-Antikörper-Reaktion mchr auslösbar. Wurde das gercinigte Membranprotein jedoch mit Erythrocyten-Phosphatiden oder synthetischen Lecithinen, die die Fettsäuren Ol- oder Linolsäure in C2-Position des Glycerins des Phosphatides enthielten, rekombiniert, dann trat bei cinem Lipidphosphorgehalt des Rekombinates von 10-18 g/kg eine positive ScriULTLDALE-Reaktion auch an Phospholipasc-bchandelten Muskelsegmenten ein. In dem Albumin-Antialbumin-System hingegen war auch ohne Kombination des Antigens mit Phosphatiden nach Phospholipasc A-Inkubation des Testmuskcls eine Anaphylaxic zu beobachten. Diesc Protcin-Phosphatid-Wechsclwirkung erscheint Voraussetzung für dic Antigen-Antikörper-Reaktion im Rh-D-System.
\end{abstract}

\section{The importance of membrane phospholipids in the antigen-antibody binding in the R/J-D-system;}

After active and passive immunization of guinen pigs, the Rh-D-antigenicity of a purified membrane protein from Rh-positive erythrocytes was demonstrated with the smooth utcrus muscle by the Scriustz-DAle method. No antigen-antibody reaction could be demonstrated after incubation of the test muscle with phospholipase A. Purified membrane protein was recombined with crythrocytcs, phosphatides, or synthetic lecithins containing oleic or linolcic acid at C-2 of the glyccrol; this a preparation containing $10-18 \mathrm{~g}$ lipid phosphorus per $\mathrm{kg}$, which produced a positive Scrultz-Dale reaction, even after treatment of the muscle segments with phospholipasc A. In the albuminantialbumin system, however, anaphylaxis was observed in the phospholipase $A$-treated test muscle, without combination of the antigen with phosphatides. This interaction of protein and phosphatide appears to be a requirement for the antigen-antibody reaction in the Rh-D-system.

In den Untersuchungen über die Rh-D-Antigenität eines kleinmolekularen Membranproteins, isoliert von Rh-positiven Erythrocyten, fanden wir, daß dieses Protein nur an Anti-D gebunden wird, wenn es mit Phospholipiden bestimmter Fettsäurezusammensetzung assoziiert ist. In dem Schultz-Dale-Test hingegen zeigte das Protein auch ohne die Phospholipid-Assoziation sowohl nach aktiver Immunisierung der Versuchstiere mit dem Protein Rh-positiver Erythrocyten als auch nach passiver Sensibilisierung des Testmuskelsegmentes mit Anti-D eine gut reproduzierbare AntigenAntikörper-Reaktion. Die Aufklärung dieser Diskrepanz in dem Antigen-Nachweis soll hier mitgeteilt werden.

\section{Methodik}

Dic Methoden zur Isolierung und biochemischen Charakterisicrung des Membranproteins wurden an anderer Stelle $(1,2,3)$ ausführlich dargestellt und sollen hiet nur insoweit erwähnt werden, wie sic für das Verständnis der nachfolgenden Untersuchungen erforderlich sind.

Der Haemagglutinations-Hemmtest wurde nach der RöhrchenMethode durchgcfïhrt, wobei das verwandtc Anti-D so cingestellt war, daß cs mit Rh-positiven papainisierten Erythrocyten gerade noch maximale Agglutination zeigte.

In dem Schultz-Dalz-Test (3) wurden folgende Immunisicrungen vorgenommen:

1. Aktive Immunisierung (4)

$200 \mathrm{~g}$ schwere weibliche Mccrschweinchen wurden im Abstand von 5 Tagen mit 10,5 und $5 \mathrm{mg}$ Protein, isoliert von Rh-positiven Erythrocyten, zusammen mit 0,5 ml FreUND'schem $\Lambda$ djuvans immunisiert. Am 20. Tag nach der ersten Injektion wurden dic Tiere unter Vermeidung von Schocksymptomen getötet und der Uterus cxstirpiert. Das Blut wurde zur Artikörper-Bestimmung durch Herzpunktion entnommen. Die Uterushörner wurden zum SchulTtz-DAle-Test verwendet, der in Tyrode-Lösung pH 7,2 bei $37^{\circ} \mathrm{C}$ unter Saucrstoffsättigung durchgeführt wurde.

2. Passive Immunisicrung in vivo (5)

Im Abstand ron $12 \mathrm{~h}$ wurden $2 \mathrm{mal} 0,5 \mathrm{ml}$ Anti-D-Scrum, Titer $1: 512$, gereinigt über DEAE Cellulose und Hydroxylapatit, $200 \mathrm{~g}$ schweren Mcerschweinchen i. p. injizicrt. Die Utcruscxstirpation und Blutentnahme erfolgte wie oben.

3. Passive Sensibilisicrung in vitro (6)

Dic Uterussegmente wurde $11 / 2 \mathrm{~h}$ in eincm Anti D-Tyrodebad, bestchend aus $0,2 \mathrm{ml}$ Anti $\mathrm{D}$, Titer $1: 512,+0,8 \mathrm{ml}$ Tyrode unter $\mathrm{O}_{2}$-Sättigung sensibilisiert und danach zum Verșuch verwendet.

4. Phospholipase-Inkubation (Phospholipase A)

Die Phospholipase A-Inkubation des Muskel-Segmentes wurde $1 \mathrm{~h}$ bei $37^{\circ} \mathrm{C}$ in $0,4 \mathrm{ml}$ Tyrodclösung, die $5 \mu \mathrm{g}$ Phospholipase A 2 pro $100 \mathrm{mg}$ Muskelfcuchtgewicht enthielt, unter ständiger Saucrstoffzufuhr vorgenommen. Hierzu wurde Phospholipase A 2 Bochringer Nr. 15057 Crolalus terr. terr. Aktivität $200 \mathrm{U} / \mathrm{ml}$ in $0,1 \mathrm{ml}$ dest. Wasser bei $2^{\circ} \mathrm{C}$ gclöst.

Für den Schultz-Dare-'Test wurde im Anschluß an dic Phospholipase A-Inkubation des Uterussegmentes mit Anti $D$ in vitro wie unter 3. scnsibilisicrt.

5. In cincr zweiten Versuchsreihe wurden parallel der passiven Sensibilisicrung des Muskelsegmentes in vitro mit Anti D mit und ohne Phospholipase $\Lambda$-Jnkubation der Testmuskel mit AntiAlbumin $0,05 \mathrm{ml}+0,5 \mathrm{ml}$ Tyrode passiv in vitto sensibilisiert.

Dic Versuchsanordnung des Schultz-Dals-Testes, die im cinzelnen bercits publiziert wurde, wurde beibehalten. Dic Muskelseginente wurden in einem $5 \mathrm{ml}$ fassenden temperierten, $\mathrm{O}_{2-}$

ग) Mit Unterstützung der Deutschen Forschungsgemeinschaft. 
gesättigten Tyrodebad bei pH 7,2 und $37^{\circ} \mathrm{C}$ mit einem Biegestab verbunden. Die konstante Muskelspannung wurde mit einem $1 \mathrm{~g}$ schweren Silbcrdraht als Gegengewicht erreicht. Während des Versuches war das Muskelbad mit einem sauerstoff-gesättigten Tyrode-Reservoir verbunden, das nach jedem Versuchsabschnitt den schnellen Wechsel der Badfüssigkeit erlaubt. Die Muskelkontraktionen wurden über den Biegestab in elektrische Impulse gewandelt, die mit Hilfe eines Schreibers registriert wurden. Nach Äquilibrierung des Testmuskels durch aufeinandetfolgende Zugabe ron $10,4,2$ und $0,2 \mu \mathrm{g}$ Histamindihydrochlorid in das Tyrodebad wurde zunächst mit nicht-korrespondierenden Antigenen eine unspezifische Kontraktion ausgeschlossen. Bei konstanter Null-Linie wurde $1 \mathrm{mg}$ korrespondierendes Antigen in das Muskelbad appliziert. Nach der durch die Antigen-AntikörperReaktion ausgelösten Muskelkontraktion wurde mit der gleichen Antigenmenge die Desensibilisierung überprüft. Nach Abschluß der Untersuchungsteihe wurde an dem gleichen Muskelsegment die Histaminempfindlichkeit mit der Histamindosis getestet, die vor der Antigen-Applikation eine optimale Muskelkontraktion ausgelöst hatte.
Folgende Antigene wurden getestet:

a) Phospholipidreiches Ausgangsmaterial, isoliert von $\mathrm{Rh}$ positiven und Rh-negativen Erythrocyten, Lipidphosphorgehalt $9 \mathrm{~g} / \mathrm{kg}$,

b) gereinigtes Rh-positives Membranprotein, Lipidphosphorgehalt $2 \mathrm{~g} / \mathrm{kg}$,

c) Protein-Phospholipid-Rekombination, Lipidphosphorgehalt $10-18 \mathrm{~g} / \mathrm{kg}$.

Die Phosphorbestimmung wurde nach der Methode von $C_{\text {HEN }} \mathrm{jr}$. et al. durchgeführt, die noch eine Phosphormenge von $1 \mu \mathrm{g}$ erfaßt.

Die Phosphatid-Identifizierung wurde auf Kieselgel-Dünnschichtplatten mit den Laufmitteln Chloroform:Methanol:Wasser (Volumina $130 \mathrm{ml}+50 \mathrm{ml}+8 \mathrm{ml}$ ) und Chloroform:Methanol: Diäthyläther: Eisessig: Wasser (Volumina $60 \mathrm{ml}+50 \mathrm{ml}+20 \mathrm{ml}$ $+20 \mathrm{ml}+15 \mathrm{ml}$ ) ausgeführt.

Die Fettsäuren wurden mit methanolischer Salzsäure verestert. Die Methylester wurden mit dem Gaschromatographen Varian 1400 auf einer Metallsäule mit den Dimensionen 2,80 × 0,5 mm i. D.,
Abb. 1

ScHULTZ-DALE-Test an Meerschweinchenuterushorn nach aktiver Immunisierung mit dem Membranprotein von Rh-positiven Erythrocyten, passiver Immunisierung mit gereinigtem Anti-D in vivo und passiver Sensibilisierung mit gereinigtem Anti-D in vitro. Korrespondierendes Antigen: Rh-positives ProHum , nichtkorrespondierende Antigene: von Rh-negativen Erythrocyten
volin
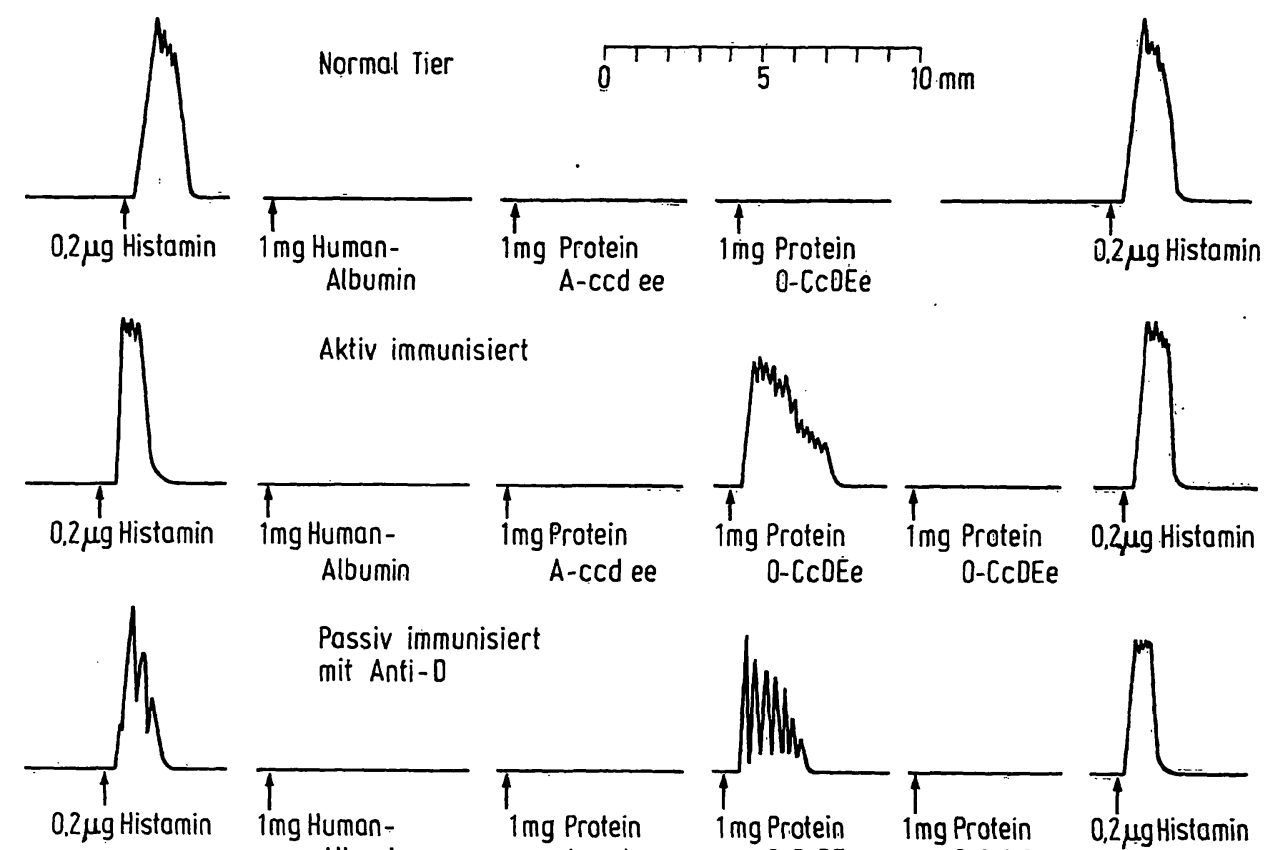

Passiv immunisiert

mit Anti-D
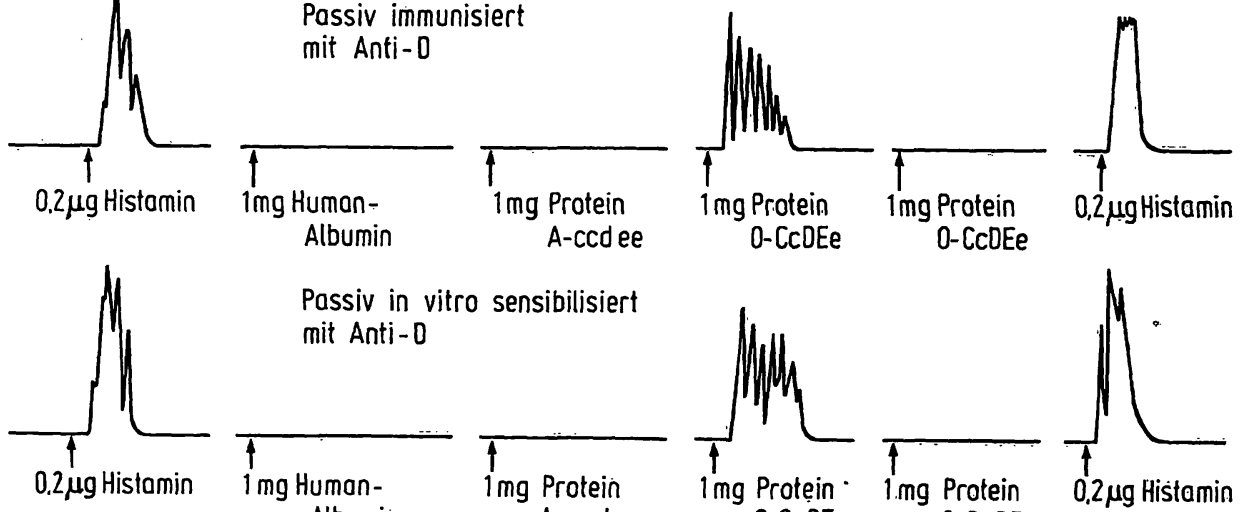

Passiv in vitro sensibilisiert mit Anti-0
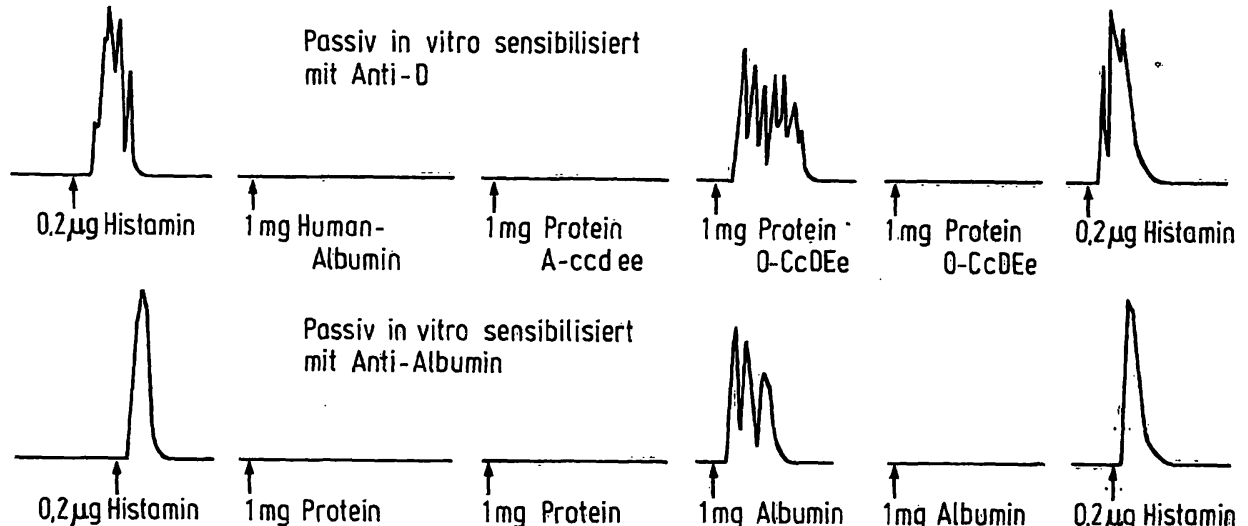

Passiv in vitro sensibilisiert mit Anti-Albumin

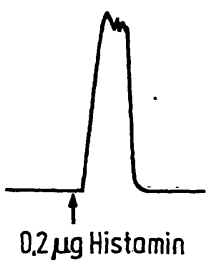

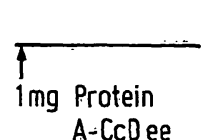
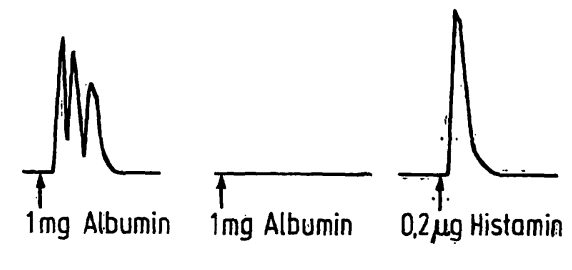

Passiv in vitro sensibilisiert mit Anti-D

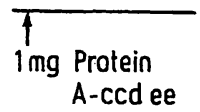

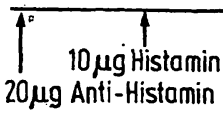

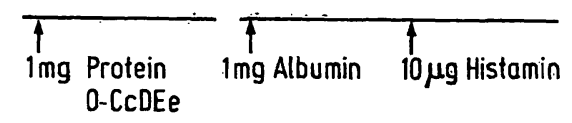

Z. Klin. Chem. Klin. Biochem. / 11. Jahrg. 1973 / Heft 12 
belegt mit $10 \%$ EGSSX auf Gaschrom P $(125-150 \mu \mathrm{m})$ bei $160^{\circ} \mathrm{C}$ isotherm aufgetrennt. Als interner Standard wurde $15 \mu \mathrm{g}$ Pentadekansäure injiziert.

Die Lipidphosphor-Verringerung der Muskelmembran durch Phospholipase A-Inkubation wurde im Anschluß an den SCFuLTzDALE-Test an den Testmuskelsegmenten bestimmt, nachdem $1 \mathrm{~h}$ bei $40^{\circ} \mathrm{C}$ mit Chloroform:Methanol $=120 \mathrm{ml}+10 \mathrm{ml}$ die Phospholipide extrahicrt worden waren. Der Lipidphosphorgehalt wurde in der Extraktionslösung gemessen und auf $100 \mathrm{mg}$ Muskelfeuchtgewicht bezogen. Die Differenz zwischen Phospholipase A-behandelten und nicht Phospholipase A-inkubierten Segmenten ergab die Lipidphosphormenge, die durch Phospholipase A-Inkubation abgespalten worden war.

\section{Protein-Pbospholipid-Rekombination}

$10 \mathrm{mg}$ gereinigtes Membranprotein von Rh-positiven bzw. Rhnegativen Erythrocyten, Lipidphosphorgehalt $2 \mathrm{~g} / \mathrm{kg}$, wurden in $0,5 \mathrm{ml}$ dest. Wasser gelöst. In $0,5 \mathrm{ml}$ wasserfreiem Äther wurden $5 \mathrm{mg}$ Phospholipid gelöst und nach $\mathrm{Zugabe}$ von $0,5 \mathrm{ml} \mathrm{H} \mathrm{H}_{2} \mathrm{O}$ $30 \mathrm{~s}$ mit $\{60 \mathrm{kHz}$ ultrabeschallt. Danach wurde die wäßrige Proteinlösung mit der phospholipidhaltigen Ätherlösung überschichtet und $2 \mathrm{~h}$ bei Raumtemperatur aufbewahrt. Der Äther wurde mit Stickstoff abgedampft. Das Phospholipid-ProteinRekombinat wurde über Sephadex $G 10$ an einer $30 \mathrm{~cm}$ langen und $0,5 \mathrm{~cm}$ weiten Säule gefiltert. Elutionsmittel Wasser; Elutions- geschwindigkeit $60 \mathrm{ml} / \mathrm{h} .1 \mathrm{mg}$ der beiden erhaltenen Fraktionen wurde zur Antigen-Bestimmung im Hämagglutinations-Hemmtest im SCHi lTZ-DaLE-Versuch eingesetzt.

\section{Ergebnisse}

Die D-Antigenität des Membranproteins von $\mathrm{Rh}$ positiven Erythrocyten, das ein Molekulargewicht von 4-5000, einen Stickstoffgehalt von $14,5 \%$, einen Lipidphosphorgehalt von $0,2 \%$ besitzt, konnte in dem SChultz-Dale-Test am Uterushornmuskelsegment des Meerschweinchens nachgewiesen werden, obwohl es in dem Hämagglutinations-Hemmtest keine Anti-D-Hemmung gezeigt hatte. Eine positive Antigen-AntikörperReaktion war sowohl nach aktiver Immunisierung mit dem Protein von Rh-positiven Erythrocyten als auch nach passiver Immunisierung des Versuchstieres in vivo und passiver Sensibilisierung des exstirpierten Uterussegmentes in vitro mit Anti $D$ bei 120 Untersuchungen reproduzierbar (Abb. 1). Lediglich in 4 Versuchen, bei denen mangelnde $\mathrm{O}_{2}$-Versorgung des Muskelbades vorgelegen hatte, trat keine adäquate

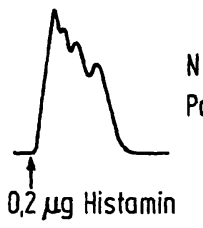

N

Passiv in vitro Anti-D

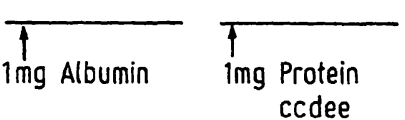

$P=0,2 \%$

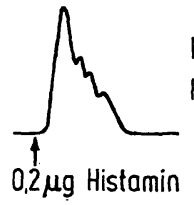

PL-A

Passiv in vitro Anti-0
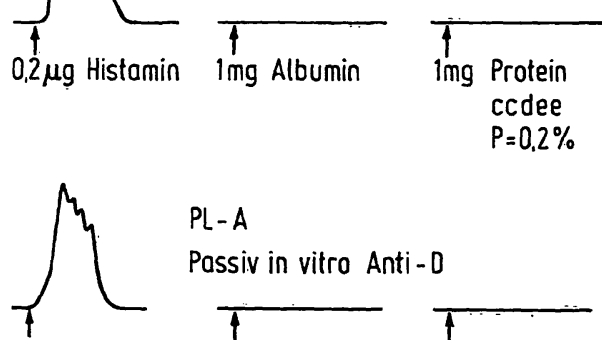

PL-A

Passiv in vitro Anti-D

$0.2 \mu \mathrm{g}$ Histomin
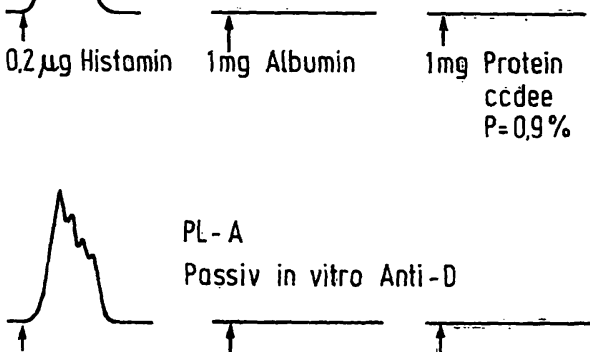

PL - A

$0,2 \mu$ Histamin

Passiv in vitro Anti-0
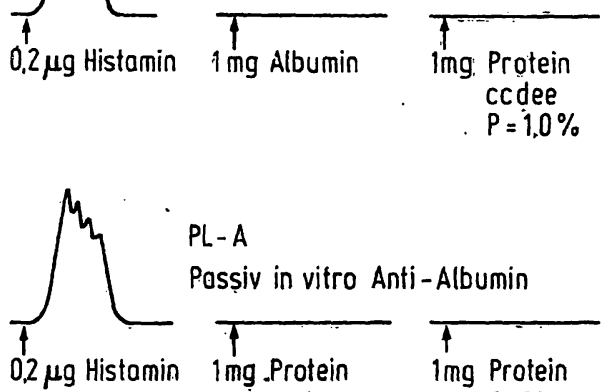

PL-A

Posșiv in vitro Anti-Albumin

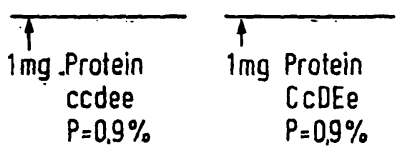

+Reinsubstanz
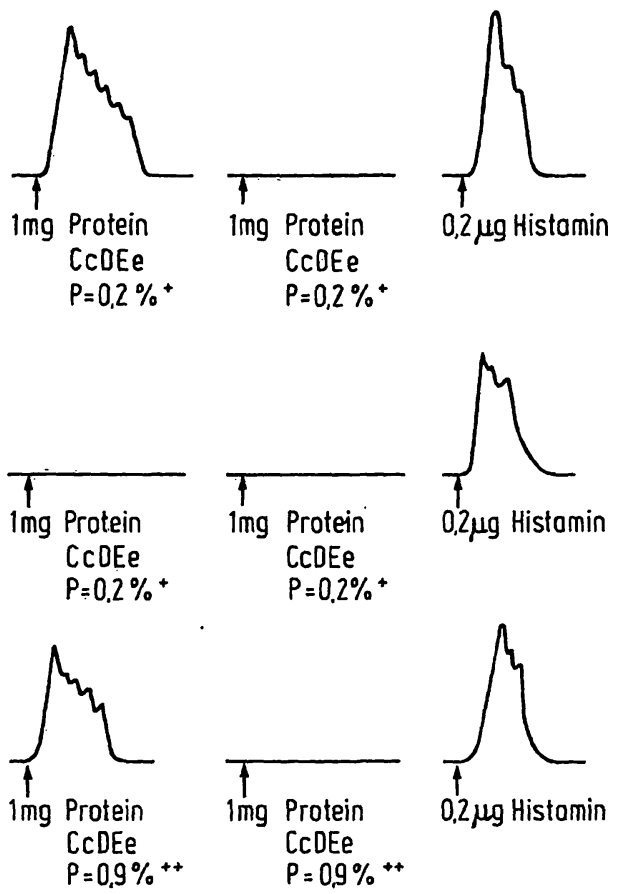

$0,2 \mu \mathrm{g}$ Histamin

$P=0.9 \%^{++}$
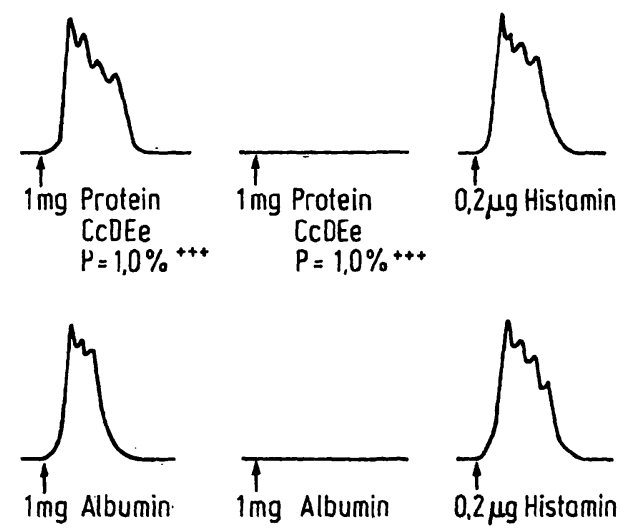

Abb. 2

SCHULTZ-DALE-Test an Meerschweinchenuterushorn mit $(N)$ und ohne $(P L-A)$ Phospholipase A-Inkubation bei passiver Sensibilisierung mit Anti-D-Vergleichsuntersuchungen im Albumin, gleichsuntersuchungen im
Anti-Albumin-System 
Tab. 1

ScHultz-DALE-Test bei Verwendung verschiedener Antigen-Arten, Bestimmung der Lipidphosphor-Abspaltung nach Phospholipase A-Inkubation des Testmuskels, Ermittlung der Lipidphosphormenge durch Chloroform: Methanol-Extraktion der Testmuskelstücke und P-Bestimmung

\begin{tabular}{|c|c|c|c|c|c|c|c|}
\hline Art der Sensibilisierung & Antigen & $\begin{array}{l}\text { Versuchs- } \\
\text { anzahl } \\
n\end{array}$ & $\begin{array}{l}\text { Lipidphosphor- } \\
\text { gehalt des } \\
\text { Antigens in } \\
\text { g/kg }\end{array}$ & $\begin{array}{l}\text { SCHULTZ-DALE } \\
\text { Reaktion }\end{array}$ & $\begin{array}{r}\text { Schultz-D } \\
\text { Reaktio } \\
\text { nach } \\
\text { Phospholip } \\
\text { A-Inkubat }\end{array}$ & $\begin{array}{l}\text { DALE } \\
\text { on } \\
\text { pase } \\
\text { tion } \\
\text { i }\end{array}$ & $\begin{array}{c}\text { Lipidphosphor- } \\
\text { verringerung nach } \\
\text { Phospholipase } \\
\text { A-Inkubation } \\
\text { des Testmuskel- } \\
\text { segments in } \%\end{array}$ \\
\hline Anti-Human-Albumin passiv in vitro & $\begin{array}{l}\text { Human- } \\
\text { Albumin }\end{array}$ & 12 & $<-1$ & 12 & 12 & & $25-30$ \\
\hline Anti-Human-Albumin passiv in vitro & Rinder-Albumin & 12 & $<-1$ & 0 & 0 & & $25-30$ \\
\hline Anti-D passiv in vitro & $\begin{array}{l}\mathrm{Rh}(+) \\
\text { Protein unge- } \\
\text { reinigt }\end{array}$ & 7 & $10-12$ & 7 & 7 & & $25-30$ \\
\hline Anti-D passiv in vitro & $\begin{array}{l}\text { Rh }(+) \\
\text { Protein ge- } \\
\text { reinnigt }\end{array}$ & 20 & $1-2$ & 20 & 0 & · & $25-30$ \\
\hline Anti-D passiv in vitro & $\begin{array}{l}\text { Rekombinat } \\
\text { Rh (+) Protein } \\
\text { gereinigtes } \\
\text { Erythrocyten- } \\
\text { Lecithin }\end{array}$ & 10 & $12-16$ & 10 & 10 & . & $25-30$ \\
\hline Anti-D passiv in vitro & $\begin{array}{l}\text { Rekombinat } \\
\text { Rh }(+) \text { Protein } \\
\text { synthetisches } \\
\text { Lecithin } C_{18} C_{18}\end{array}$ & 3 & $10-14$ & 3 & 0 . & . & $25-30$ \\
\hline $\begin{array}{r}\text { Anti-D passiv in vitro } \\
\qquad:\end{array}$ & $\begin{array}{l}\text { Rekombinat } \\
\text { Rh }(+) \text { Protein } \\
\text { synthetisches } \\
\text { Lecithin } C_{16} C_{18,2}\end{array}$ & 3 & $10-16$ & 3 & 3 & & $25-30$ \\
\hline Anti-D passiv in vitro & $\begin{array}{l}\text { Rh }(\rightarrow \text { Protein } \\
\text { ungereinigt }\end{array}$ & 10 & $10-12$ & 0 & 0 & & $25-30$ \\
\hline Anti-D passiv in vitro & $\begin{array}{l}\text { Rekombinat } \\
\text { Rh }(\rightarrow \text { Protein } \\
\text { gereinigt } \\
\text { Erythrocyten- } \\
\text { Lecithin }\end{array}$ & 5 & $10-16$ & 0 & 0 & & $25-30$ \\
\hline
\end{tabular}

Muskelkontraktion ein. Phosphatide von Rh-positiven und Rh-negativen Erythrocyten, Proteine von $\mathrm{Rh}$ negativen Exythrocyten und andere nicht-korrespondierende Antigene wie Humanalbumin oder Rinderalbumin führten zu keiner anaphylaktischen Reaktion. Antihistaminica verhinderten die Antigen-AntikörperReaktion.

Muskelsegmente, die vor der Sensibilisierung mit Anti D in vitro mit Phospholipase A 2 inkubiert worden waren, zeigten nach Applikation von $2 \mu \mathrm{g}$ Histamindihydrochlorid noch eine gute Muskelkontraktion. Mit' dem gereinigten Membranprotein, dessen Phospholipidgehalt $2 \mathrm{~g} / \mathrm{kg}$ betrug, war jedoch nach Phospholipase AInkubation des Testmuskels keine positive SchulrzDALE-Reaktion auszulösen. Hingegen konnte mit der Phospholipid-reichen Ausgangssubstanz und mit den Protein-Phospholipid-Rekombinaten, bestehend aus dem gereinigten Rh-positiven Protein und ErythrocytenLecithin oder-Kephalin eine sichere anaphylaktische Reaktion erhalten werden. In gleicher Weise provozierte auch das Rekombinat aus dem gereinigten Membranprotein mit synthetischen Lecithinen, die in C 2-Position entweder die ungesättigten Fettsäuren Öl- oder Linolsäure enthielten, eine Muskelkontraktion mit Desensibilisierung. Hingegen trat keine positive Schultz-Dale-Reaktion mit dem Protein-DipalmitinLecithin-Rekombinat ein. Voraussetzung für eine positive Schultz-Dale-Reaktion war ein Phosphor- gehalt von mindestens $10-15 \mathrm{~g} / \mathrm{kg}$ und die Gegenwart der ungesättigten Fettsäuren in C2-Position des Glycerins von Lecithin oder Kephalin. Im Gegensatz zu dem Membranprotein Rh-positiver Erythrocyten konnte mit Humanalbumin auch nach Phospholipase ABehandlung des Testmuskels bei passiver Sensibilisierung des Testmuskels mit Anti-Albumin in vitro eine Antigen-Antikörper-Reaktion erhalten werden, ohne daß eine Phospholipid-Kombination des Antigens durchgeführt worden war (Abb. 2).

Die Protein-Phospholipid-Rekombinate des Rh-positiven Proteins, die an Phospholipase A behandelten Muskelsegmenten eine Anaphylaxie ausgelöst hatten, hemmten Anti-D im Hämagglutinations-Hemmtest mit 3-4 Titerstufen. Die Phospholipid-Verringerung durch Phospholipase A-Inkubation des Testmuskels betrug 25-30\% der mit Chloroform-Methanol extrahierbaren Phospholipide (Tab. 1).

\section{Diskussion}

Da mit dem gereinigten Membranprotein im SchuLtzDALE-Test nach aktiver und passiver Immunisierung eine gut reproduzierbare Antigen-Antikörper-Reaktion zu erzielen war, ist anzunehmen, daß dieses Protein für die $\mathrm{D}$-Antigenität im $\mathrm{Rh}$-System verantworlich ist. Bei der Antigen-Antikörper-Bindung ist jedoch die Gegenwart eines Phospholipides notwendig, da nach Phospholipase-Behandlung des Testmuskels mit gereinigtem 
Rh-positiven Protein keine Schultz-Dale-Reaktion mehr auslösbar war. Hingegen trat nach Rekombination dieses Proteins mit Lecithin oder Kephalin von Rhpositiven oder Rh-negativen Erythrocyten auch an dem Phospholipase A behandelten Muskelsegment eine Anaphylaxie ein. Die Protein-Phospholipid-Rekombination, über die an anderer Stelle ausführlich berichtet werden soll, führte nur zu einer Antigen-AntikörperReaktion, wenn in C 2-Position des Glycerins des Phospholipides die ungesättigten Fettsäuren Öl- oder Linolsäure vorlagen. und der Lipidphosphorgehalt höher als $10 \mathrm{~g} / \mathrm{kg}$ war. Die Bedeutung dieser ungesättigten Fettsäuren konnten wir durch synthetisches Lecithin zeigen, bei dem das Rekombinat mit Dipalmitin-Lecithin keine Anaphylaxie auslöste, hingegen synthetische Lecithine mit Öl- oder Linolsäure in C 2Position des Glycerins des Lecithins eine eindeutige Antigen-Antikörper-Reaktion zeigten. Aufgrund dieser Befunde ist anzunehmen, daß die für die AntigenAntikörper-Bindung erforderliche Proteinkonformation durch adäquate Phospholipide induziert werden kann. In dem Schultz-Dale-Test übernehmen Phospholipide der Muskelmembran die Rolle der Erythrocytenmembran-Phospholipide, șo daß auch das gereinigte Protein bereits eine Antigen-Antikörper-Reaktion auslöst. $\mathrm{Da}$ sowohl Phospholipide von Rh-positiven als auch von $\mathrm{Rh}$-negativen Exythrocyten zur erfolgreichen Rekombination verwendet werden konnten, ist es unwahrscheinlich, daß Phospholipiden eine spezifische AntigenEigenschaft zukommt. Diese Befunde entsprechen den Ergebnissen von GreEN $(8,9,10)$, der fand, daß Rhpositives Erythrocytenstroma nach Butanol- oder Chloroform-Methanol-Extraktion kein Anti-D mehr bindet. Erst nach Rekombination des extrahierten Rhpositiven Stromas mit Phospholipiden, unabhängig davon, ob sie von $\mathrm{Rh}$-positiven oder $\mathrm{Rh}$-negativen Erythrocyten gewonnen worden waren, konnte die Rh-D-Antigenität des Stromas bis zu $75 \%$ wieder erhalten werden. Hierbei zeigte Lecithin einen besseren Effekt als Kephalin. Da wir in dem Albumin-AntiAlbumin-System auch an Phospholipase A-behandelten Muskelsegmenten ohne Phospholipid-Kombination des Antigens eine positive SCHULTZ-DALE-Reaktion erhielten, ist anzunehmen, daß die Gegenwart der Phospholipide nur für das D-Anti-D-System entscheidend ist. Analog zu anderen Phospholipid-Protein-Komplexen ist es deshalb zu diskutieren, ob die AntigenAntikörper-Bindung im Rh-System von dem $\alpha$-HelixGehalt des Proteins abhängig ist, der durch Phospholipide begünstigt werden kann. Durch circulardichrographische Messungen vor und nach der ProteinPhospholipid-Rekombination soll diese Frage der Protein-Konformation, die zur optimalen Anti-D-Bindung führt, experimentell untersucht werden.

Die Diskrepanz in dem Rh-D-Antigen-Nachweis $z$ wischen Schultz-Dale-Test und HämagglutinationsHemmtest ist aufgrund der vorliegenden Ergebnisse folgendermaßen zu deuten: In dem Schultz-DaleVersuch erhält das gereinigte $\mathrm{Rh}$-positive Protein durch die adäquaten Phospholipide der Muskelmembran die für die Antigen-Antikörper-Reaktion erforderliche Konformation. In dem Hämagglutinations-Hemmtest hingegen haben die zellständigen $\mathrm{Rh}$-Antigene der Testerythrocyten durch die benachbarten Membranphospholipide bereits die optimale Konformation, die für die Anti D-Bindung notwendig ist. Sie sind deshalb dem isolierten gereinigten Protein, das keine adäquaten Phospholipide besitzt, überlegen, da das Phospholipidfreie Protein nicht in der für die Antikörper-Bindung erforderlichen Konformation vorzuliegen scheint.

\section{Danksagung}

Den technischen Assistentinnen Frau INGEBORg ENGLERT und Fräulein MARGot BürKLE danken wir für die wertvolle technische Unterstützung bei Durchführung der Isolierung des Membranproteines und der Antigen-Austestung.

\section{Literatur}

1. Weicker, H., KRopp, J., Metz, J., Ritthaler, F. \& Ebert, W. (1972), Clin. Chim. Acta 37, 59-70. - 2. WeICKER, H., KROPP, J. \& ROELCKE, D. (1971), diese Z. 9, 375-382. - 3. Dale, H. H. (1912), J. Pharm. Exp. Ther. 2, 221. - 4. Coulson, E. J. (1953), J. Allergy 24, 458-473. - 5. Kropp, J. (1970), Dissertation Heidelberg. - 6. HALPERN, B. N., Lracopoulos, P.,
Liacopolous-Briot, M. \& Binaghi, R. (1959), Immunology 2, 351 - 362. - 7. C HEN, S. P. jr., ToribarA, F. Y. \& WARnER, H. (1956), Anal. Chem. 28, 1756-1759. - 8. GreEN, F. A. (1965), Vox Sang. 10, 32-53 - 9. Green, F. A. (1968), J. Biol. Chem. 243, 5519-5521. - 10. GreEN, F. A. (1972), J. Biol. Chem. 247, $881-887$.
Prof. Dr. H. Weicker 69 Heidelberg Hospitalstr. 3 